\title{
Dynamical Approach to Manipulation of Single Atoms/Molecules at Material Surface
}

\author{
Takashi Hikihara
}

\begin{abstract}
Nano-technology is a bottom-up technology and shows the possibility of spatial arrangement of atoms and molecules by using sensing probes, for example, SEM, AFM, and so on. The demonstrative experiments have been reported since 1990s. In this paper, we will discuss a model of single particles manipulation from material surface. The model is based on a van der Waals molecule vibrational predissociation of T-shaped model. The probability of the manipulation is also considered with relation to chaotic dynamics. The theoretical discussion is confirmed by numerical simulation. Through the results, the method for manipulation of nano-particles is discussed.
\end{abstract}

\section{INTRODUCTION}

This paper describes the dynamics of single atoms and molecules, bonding to material surface by van der Waals force, at one of potential wells under external excitation.

Recently, manipulation of single atoms and molecules has been experimentally achieved on surfaces with use of scanning tunneling microscope (STM) and atomic force microscopy (AFM) in lateral processes as well as vertical processes [1], [2], [3], [4], [5]. The lateral processes can transfer atoms and molecules parallel to the surface and the vertical between tip and adatom via vibrational excitation of the target-substrate bond. The processes have been also studied theoretically [6], [7], [8], [9] and numerically [10].

This paper applies van der Waals molecular vibrational predissociation based on T-shaped model [11], [12], [13], [14], [15]. The model was introduced to describe a quantum mechanics that governs the rates of vibrational predissociation of A-B-A triatomic molecules, which are coupled Morse oscillators. Atoms and molecules attached on the material surface are bonded by van der Waals potential, which is characterized by Morse type atom-atom interaction potential. The dynamics of manipulated particles is described by the fragmentation of the atom B from the coupling of AA.

In the following section, we introduce a Hamiltonian of the triatomic molecules. The system is coupled nonlinear oscillators. Assuming that the rotation and bending modes are neglected, at first, it is shown that the eigen frequency decides the resonance and energy exchange. At high energy, the system possibly shows chaotic vibration. It gives the probability to classical dynamics of atoms and molecules. The fragmentation of atoms from surface is discussed based

This work was partially supported by GCOE program.

T.Hikihara is with Department of Electrical Engineering, Kyoto University, Katsura, Nishikyo, Kyoto 615-8510, Japan and Photonics and Electronics Science and Engineering Center, Kyoto University, Katsura, Nishikyo, Kyoto 615-8510, JAPAN hikihara@kuee.kyoto-u.ac.jp on the global phase structure. We also confirm the possibility of manipulation by numerical simulation. When the perturbation of energy exceeds the critical value, the dynamics appears the global behavior out of single potential well. That is, the vibratory dissociation can achieve the manipulation of nano-particles. This is inevitable to capture the atom dissociated from the material surface. The possibility of capture is also discussed based on the setting of dissipation. The results show us the possible manipulation method of nano-particles in situ conditions.

\section{Dynamics of Single Atom at Material Surface}

In this section, the dynamics of single atoms is discussed based on Hamiltonian mechanics. At first we introduce a model of atoms and molecules alignment for estimating fragmentation of atom from surface bond.

\section{A. Model of Single Atom and Molecule}

Here we focus on the dynamics of single atoms and molecules attached on material surface at low temperature and vacuum circumstances. Assume that single atoms or molecules B at a surface is bonding to quadri-atoms A (see Fig.1). The model of molecular vibrational fragmentation has been discussed for T-shaped structure with van der Waals potential. At the surface, we assume the pyramid structure of atoms at steady state. In the pyramid-shape bonding, the rotation and bending modes disappear. Then the DOF (degree of freedom) of B is restricted in the vertical direction to the rectangle plane of $\mathrm{A}$. Hereafter, the system can be modeled by T-shaped structure with diagonal atoms A and B without rotating dynamics.

The Morse interaction potential limits the distance of the interaction in a short range. In the region, the atoms are called Velet neighbors [16]. The distance between the atoms $\mathrm{A}$ is depicted as $\sqrt{2} Q$ and distance between $\mathrm{B}$ and center $\mathrm{O}$ of A-plane as $q$, which is also on the axis. Then the distance between $\mathrm{A}$ and $\mathrm{O}$ becomes $Q$, which is perpendicular to
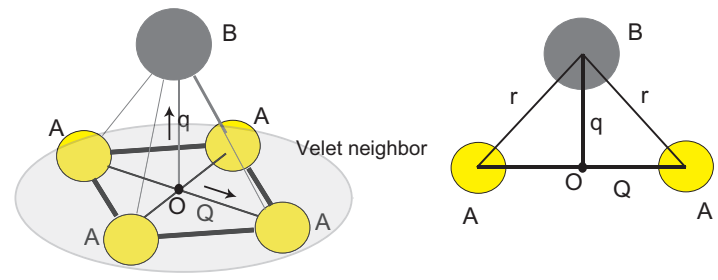

Fig. 1. Atoms and molecules alignment at material surface. 


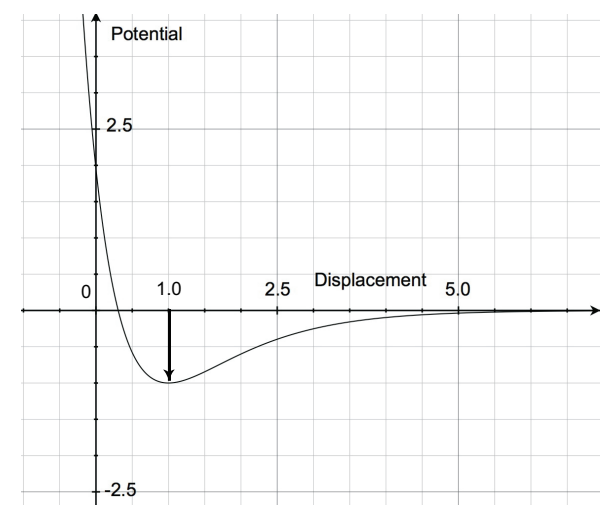

Fig. 2. Schematic Morse potential with equilibrium point at 1.0.

the $q$-axis. Each momentum is given by $P$ and $p$. The Hamiltonian can be written in the form:

$$
H=\frac{P^{2}}{2 m}+\frac{p^{2}}{2 \mu}+V_{0}(Q, q)+V_{1}(Q, q),
$$

where the angle between the axis of A-plane and the vector from the center of mass to $\mathrm{B}$ is restricted at rectangle. Then we neglect the kinetics of angular motion. Here $m_{A}$ and $m_{B}$ are the masses of $\mathrm{A}$ and $\mathrm{B}$, respectively. $m=m_{A} / 2$ is the reduced mass of $\mathrm{A}$ and $\mu=2 m_{A} m_{B} /\left(m_{A}+2 m_{B}\right)$ the reduced mass of the whole system. The potentials are given as follows [15]:

$$
V_{0}(Q, q)=W_{0}(Q)+W_{0}(q)
$$

where

$$
\begin{gathered}
W_{0}(Q)=D_{0 \mathrm{~A}}\left(e^{-2 \beta_{0 \mathrm{~A}}\left(Q-Q_{0}\right)}-2 e^{-\beta_{0 \mathrm{~A}}\left(Q-Q_{0}\right)}\right), \\
W_{0}(q)=D_{0 \mathrm{~B}}\left(e^{-2 \beta_{0 \mathrm{~B}}\left(q-q_{0}\right)}-2 e^{-\beta_{0 \mathrm{~B}}\left(q-q_{0}\right)}\right),
\end{gathered}
$$

and

$$
V_{1}(Q, q)=W_{1}\left(r_{+}\right)+W_{1}\left(r_{-}\right)
$$

where

$$
W_{1}\left(r_{ \pm}\right)=D_{1}\left(e^{-2 \beta_{1}\left(r_{ \pm}-r_{ \pm 0}\right)}-2 e^{-\beta_{1}\left(r_{ \pm}-r_{ \pm 0}\right)}\right) .
$$

$V_{1}$ is the expansion of van der Waals potential in the Tayler series around equilibrium point. $r_{ \pm}$are the distance between B and one of the atoms A. $r$ is a function of $Q$ and $q$. The equilibrium point is given by $r_{0} . D_{0 \mathrm{~A}}, D_{0 \mathrm{~B}}$, and $D_{1}$ depict the dissociation energy. $\beta_{0 \mathrm{~A}}, \beta_{0 \mathrm{~B}}$, and $\beta_{1}$ denote the range parameters. The shape of Morse potential is shown in Fig.2

Assuming the 2-dimensional symmetry of quadri-atoms A, the potential function around the single atom B possesses the axial symmetric property at steady state. Then, the system seems to be equivalent to T-shaped model. It implies that the fragmentation is limited in the vertical direction to the plane A.

Equation 1 represents the model system Hamiltonian in coordinates $(Q, q, P, p)$. In the classical dynamics, we have the relation:

$$
\left\{\begin{aligned}
\dot{Q} & =\frac{\partial H}{\partial P} \\
\dot{q} & =\frac{\partial H}{\partial p}, \\
\dot{P} & =-\frac{\partial H}{\partial Q} \\
\dot{p} & =-\frac{\partial H}{\partial q}
\end{aligned}\right.
$$

The linearization of the system (2) is based on Taylor expansion around the equilibrium point for $Q$ and $q$.

\section{B. Analysis based on Action-Angle Formulation [14]}

Under the dissipative or excited state, the perturbed Hamiltonian is given as

$$
H=H_{0}+\varepsilon H_{1},
$$

where $\varepsilon$ is a small parameter. $H_{1}$ includes $V_{1}(Q, q)$. The zerooder component of Hamiltonian is given by

$$
H_{0}=\frac{P^{2}}{2 m}+\frac{p^{2}}{2 \mu}+W_{0}(Q)+W_{0}(q)
$$

We can rewrite $H_{0}$ by an action( $(I)$-angle $(\theta)$ form. Based on the reference [14], the following relationship is obtained.

$$
H_{0}=E_{A}\left(I_{A}\right)+E_{B}\left(I_{B}\right)
$$

$I_{A}$ and $I_{B}$ are action variables, which are obtained by action integral. The potential energy of Morse oscillators, $E_{A}$ and $E_{B}$, are defined as follows:

$$
\left\{\begin{array}{l}
E_{\mathrm{A}}\left(I_{\mathrm{A}}\right)=\left(I_{\mathrm{A}}+\frac{1}{2}\right) \omega_{\mathrm{A}}-\left(I_{\mathrm{A}}+\frac{1}{2}\right)^{2} \omega_{\mathrm{A}}^{2} / 4 D_{0 \mathrm{~A}}-D_{0 \mathrm{~A}}, \\
E_{\mathrm{B}}\left(I_{\mathrm{B}}\right)=\left(I_{\mathrm{B}}+\frac{1}{2}\right) \omega_{\mathrm{B}}-\left(I_{\mathrm{B}}+\frac{1}{2}\right)^{2} \omega_{\mathrm{B}}^{2} / 4 D_{0 \mathrm{~B}}-D_{0 \mathrm{~B}},
\end{array}\right.
$$

where

$$
\begin{aligned}
& \omega_{\mathrm{A}}=\left(2 D_{0 \mathrm{~A}} \beta_{0 \mathrm{~A}}^{2} / m\right)^{1 / 2}, \\
& \omega_{\mathrm{B}}=\left(2 D_{0 \mathrm{~B}} \beta_{0 \mathrm{~B}}^{2} / \mu\right)^{1 / 2} .
\end{aligned}
$$

$D_{0 A}$ and $D_{0 B}$ are defined by related Morse potentials. The simple derivatives of $E_{A}$ and $E_{B}$ give the zero order frequencies in each motion along $Q$ and $q$. That is,

$$
\left\{\begin{array}{l}
\dot{\theta}_{\mathrm{A}}=-\omega_{\mathrm{A}}+\left(2 I_{\mathrm{A}}+1\right) \omega_{\mathrm{A}}^{2} / 4 D_{0 \mathrm{~A}}=\Omega_{\mathrm{A}}, \\
\dot{\theta}_{\mathrm{B}}=-\omega_{\mathrm{B}}+\left(2 I_{\mathrm{B}}+1\right) \omega_{\mathrm{B}}^{2} / 4 D_{0 \mathrm{~B}}=\Omega_{\mathrm{B}} .
\end{array}\right.
$$

The energy at which the atom $\mathrm{B}$ is separated at infinity depends on $D_{0 B}$. The external energy input for fragmentation is due to the boundary of trapped motion of $H$. The phase structure is schematically described in Fig.3.

The maximum values of $I_{A}$ and $I_{B}$ are obtained by Eq.(4) [18].

$$
\left\{\begin{array}{l}
I_{\mathrm{A} \max }=-\frac{1}{2}+\frac{2 D_{0 \mathrm{~A}}}{\omega_{\mathrm{A}}}, \\
I_{\mathrm{B} \max }=-\frac{1}{2}+\frac{2 D_{0 \mathrm{~B}}}{\omega_{\mathrm{B}}} .
\end{array}\right.
$$

These are also limits of actions for T-shaped structure in Hamiltonian. That is, the external energy input to the system can dissociate the bonding between atoms $\mathrm{A}$ and $\mathrm{B}$. 


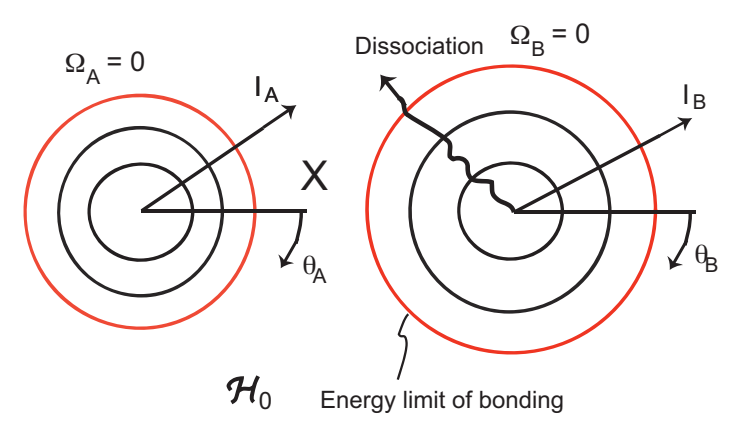

Fig. 3. Phase portrait of Hamiltonian.
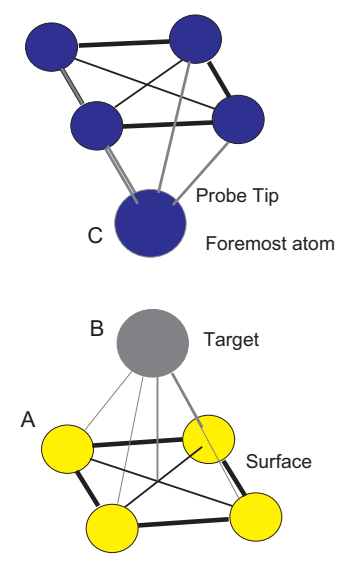

Fig. 4. Manipulation of single atom by probe.

\section{Manipulation of Single Atoms by Probe}

One of the important topics of nanotechnology is the manipulation of single atoms at the material surface. We have already known the dynamics of single atoms, which dissociate from material surface. Here, we will discuss a mathematical formulation of vibratory fragmentation of single atoms by probe based on perturbed Hamiltonian system.

\section{A. Dynamics of Atoms between Surface and Probe}

The manipulation of single atoms has been achieved by STM and AFM [1], [2], [3], [4], [5]. The schematic structure is described in Fig.4.

The manipulation implies the energy exchange between an atom bonding to surface and probe. The manipulation of atoms is governed by the probability of dissociation of atomic bond. However, the dynamics and probability have not been well understood [19]. If the probe is rigid or consists of heavy atoms with strong bond to bulk, the dynamics is simply modeled by T-shaped configuration of atoms and molecules at surface. Then, the vibration of probe gives us an external energy input to the original Hamiltonian system.

\section{B. Probability of Hamiltonian System}

The dynamics of single atoms, which are manipulated by probe, is explained with relation to the bond in Tshaped structure. The nonlinear resonances under external excitation are related to energy transfer between modes

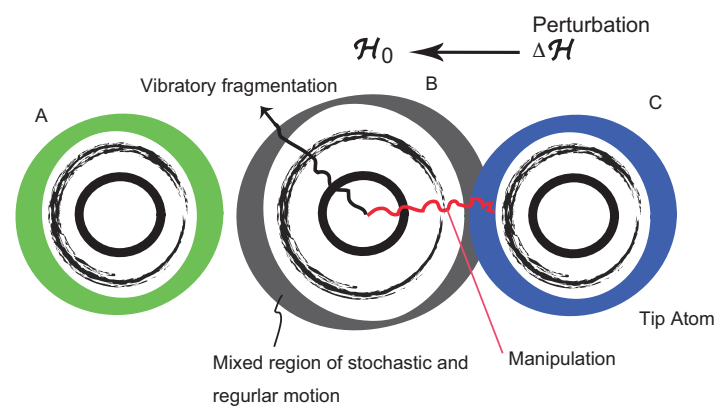

Fig. 5. Phase portrait of perturbed Hamiltonian system.

and external vibration. Moreover, the limit of resonance depicts the appearance of instability depending on the nonlinearity. On the other hand, we know that there is chaotic region around resonant boundary [13], [14], [15]. Under the external Hamiltonian perturbation $\Delta H$ might generate vibratory fragmentation and manipulation of atoms as shown in Fig.5. Therefore, the instability of the resonance around the boundary is strongly related to the global phase structure. When the system becomes dissipative, the complexity is due to the intersection of stable and unstable manifolds. Bonding instabilities are also reported between atoms at material surface and the foremost tip of probe [20]. The instability does not generate the probability but the abrupt jump of dynamics. This might be related to the uncertainty in capture and release of atoms.

In the classical treatment, the probability of fragmentation is defined by the rate of initial conditions from which trajectories come out of the region surrounded by homoclinic orbit. Consider initial conditions in the space $\left(Q_{i}, q_{i}, P_{i}, p_{j}\right)$; $i$ and $j$ show the indexes of meshed initial condition space. Their Hamiltonian trajectories are generated by

$$
\left\{\begin{array}{l}
\frac{P_{i}^{2}}{2 m}+W_{0}\left(Q_{i}\right)=E_{\mathrm{A}}\left(I_{\mathrm{A}}\right), \\
\frac{p_{j}^{2}}{2 \mu}+W_{0}\left(q_{j}\right)=E_{\mathrm{B}}\left(I_{\mathrm{B}}\right) .
\end{array}\right.
$$

These equations possibly show the stochastic region in initial condition space [14]. The dissipation to the coupled Morse oscillators loosens the homoclinic orbit corresponding to the energy limit of bonding. At the same time, the homoclinic intersection and folded manifolds appear in the global phase structure. In the case, the probability is strongly governed by the structure in spite of the uncertainty of Hamiltonian system [15].

\section{Energy Input for Manipulation}

When the forcing of probe vibrates the single atoms, the dynamics can be approximated by Eq.(2) with dissipation and forcing terms. To achieve the vibratory fragmentation, the external energy must be as much as

$$
\Delta E_{\mathrm{B}} \geq E_{\mathrm{B}}\left(I_{\mathrm{B} \max }\right)-E_{\mathrm{B}}\left(I_{\mathrm{B} 0}\right),
$$




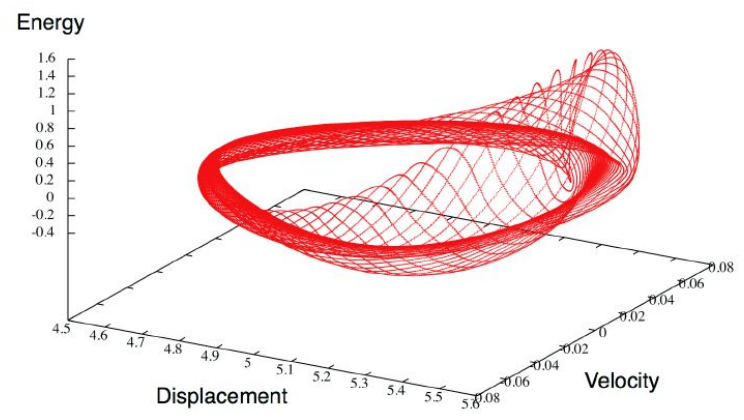

Fig. 6. Energy of manipulated dynamics at small perturbation. The dynamics is sampled at period of external forcing.

where $I_{\mathrm{B} 0}$ is the initial action value. At the same time the atom $B$ must be captured in the bond of probe tip. As for the foremost atom $(C)$ of tip, the situation coincides with energy exchange between $\mathrm{A}-\mathrm{B}$ and $\mathrm{B}-\mathrm{C}$ in the bond $\mathrm{A}-\mathrm{B}-\mathrm{C}$. After the fragmentation of the bond $\mathrm{A}-\mathrm{B}$, the atom $\mathrm{B}$ generates the new bonding $\mathrm{B}-\mathrm{C}$. In this process, the dissipation works for the stabilization of dynamics. Between release and capture, chaotic dynamics cannot be avoided as mentioned above. Then, the manipulation is governed by the uncertainty of dynamics.

\section{NUMERICAL DISCUSSION}

The manipulation dynamics of single atom between the surface and probe has already described by the Hamiltonian system. Then, we confirmed the statistic behavior of the particle. In the following numerical discussion, the parameters are set as follows: $m_{A}=m_{B}=1.0 \times 10^{-12}, D_{0 \mathrm{~A}}=D_{0 \mathrm{~B}}=$ $1.0 \times 10^{-13}$, and $\beta_{0 \mathrm{~A}}=\beta_{0 \mathrm{~B}}=2.0$. The equilibrium points are at 2.0 and 7.0 between the surface and the probe normalized by the Morse equilibrium point. Fig.6 is the periodical motion of atom. The excitation induces the disturbance of potential. The quasi-periodic behavior depends on the natural frequency of atom and forcing frequency.

On the other hand, Fig. 7 shows the change of the energy depending on the irregular trajectory. The external forcing by probe gives the disturbance of interaction force through Morse potential. When the amplitude of the forcing exceeds the critical value, the trajectory becomes irregular as shown in the figure. However, the system keeps the features of Hamiltonian system.

The motion of single atom is kept in the potential well under a weak excitation. This is the case of harmonic oscillators. Depending on the initial given energy, the trajectory shows the different closed orbit. Based on the previous discussion, the manipulation of atom requires the perturbation in energy to exceed the critical value.

The Hamiltonian system has no dissipative terms after the

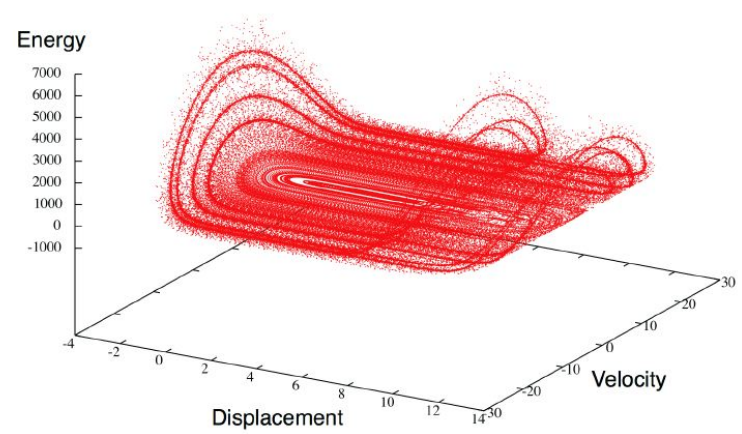

Fig. 7. Energy of manipulated irregular dynamics at the perturbation exceeding a critical value. The dynamics is sampled at period of external forcing.

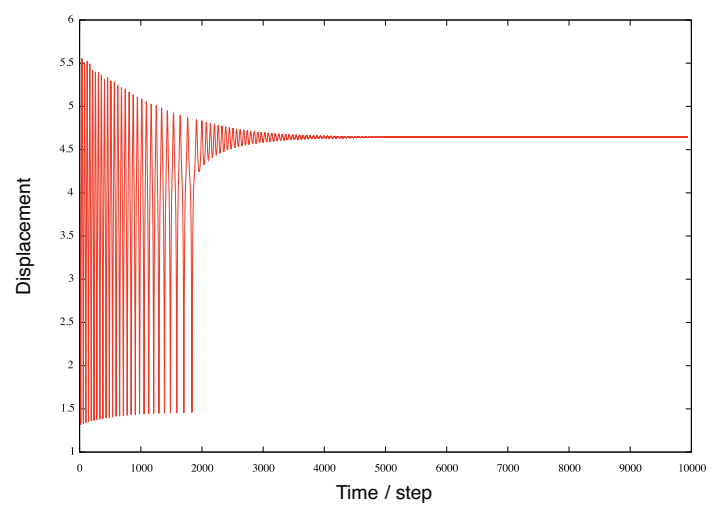

Fig. 8. Capture of nano-particle by dissipation. The dissipation is set for the displacement over 4.0 near the probe.

trajectory jumps out of the potential well. The manipulation of single atom should set the dissipative control of dynamics.

\section{Method for Manipulation}

The manipulation of nano-particles is a challenging topic. If the concept is in the Hamiltonian formulation, the idea is far from the practical setups. One of the practical considerations is the dissipation control to capture the particle. If the dissipation can be changed in the gap, the dynamics of nano-particle will be affected by the energy dissipation. Fig. 8 shows the numerical simulation of capturing a particles after irregular motion.

The atom $\mathrm{B}$ is wandering in the phase space because of the irregular motion under excitation. The dissipation is additionally given when the displacement is larger than a critical value, which is set at 4.0 in proportion to the velocity as a damping. Physically, the dissipation seems to appear as a thermal dynamics between probe and surface. In the low temperature and vacuum condition, the multibody effect might govern the thermal effect in the probe and material. It finally causes the thermal effect of the 
probe-surface system. The estimation of the effect decides the method of control. In the simulation, it clearly shows the convergence of the trajectory to the well of the probe potential. When the trajectory become chaotic, the control method based on the controlling chaos has a potential to stabilize the particle behavior. The method for controlling chaos in AFM dynamics might play an important role [21].

If there are several probes, the particle is possibly transferred between probes. The concept has already been proposed by authors [10], [22]. The design of the system is our next project.

\section{CONCLUding Remarks}

This paper discusses the manipulation dynamics of single atoms and molecules by probe at material surface along the theory of classical mechanics of vibrational fragmentation for T-shaped molecules. The Hamiltonian approach can estimate the minimum energy that corresponds to fragmentation of single atoms from other bond. At the material surface, the model is effective in low dimensional approximation through summing Velet neighbors. We showed that the formulation could describe the manipulation dynamics by vibrating probe at the material surface. In the model, the dynamics was focused on the new bond between a targeting atom and a foremost atom of tip. The dynamics also has chaotic behavior based on the nonlinearity of interaction of atoms under Morse potential. These situations are numerically confirmed in the system with external excitation of potential. It clearly shows the appearance of irregular behavior under the external excitation exceeding the critical energy level. The chaotic sea in phase space of the control variables decides the probability of manipulation.

In order to capture an atom by probe, it is inevitable to stabilize the dynamical motion jumping out of a potential well of material surface. Here, the numerical discussion shows that the key is the dissipation of energy in the system. The method for describing dissipation and its design are also important topics in the nano-dynamics. We hope that this approach can contribute to the dynamical design of nano-technology and application of nonlinear dynamics in practical systems.

\section{ACKNOWLEDGMENTS}

The author would like to show his acknowledge to Dr. Yoshihiko Susuki for his wonderful suggestion. He also acknowledges the fruitful discussion with Professor Igor Mezic, University of California, Santa Barbara for the nonlinear dynamics of nano-science.

\section{REFERENCES}

[1] D. M. Eigler and E. K. Schweizer, "Positioning single atoms with a scanning tunnelling microscope," Nature, vol.344, pp.524-526, 1990.

[2] L. Bartels, G. Meyer, and K. -H. Rieder, "Basic Steps of Lateral Manipulation of Single Atoms and Diatomic Clusters with a Scanning Tunneling Microscope Tip," Phy. Rev. Lett., 79, pp.697-700, 1997.

[3] J. A. Stroscio and R. J. Celotta, "Controlling the Dynamics of a Single Atom in Lateral Atom Manipulation," Science, 306, pp.242-247, 2004.

[4] N. Oyabu et al., "Single Atomic Contact Adhesion and Dissipation in Dynamic Force Microscopy," Phy. Rev. Lett., 96, 106101, 2006.
[5] Y. Sugimoto et al., "Mechanism for Room-Temperature Single-Aton Latteral Manipulations on Semiconductors using Dynamic Force Microscopy," Phy. Rev. Lett., 98, 106104, 2007.

[6] X. Bouju, C. Joachim, and C. Girard, "Single-atom motion during a lateral STM manipulation," Phy. Rev. B, R7845, 1999.

[7] N. Lorente and M. Persson, "Theoretical aspects of tunneling-currentinduced bond excitation and breaking at surfaces," The Royal Soc. Chem., Faraday Disscuss., 117, pp.277-290, 2001.

[8] A. Kuhnle, et al., "Understanding atom movement during lateral manipulation with the STM tip using a simple simulation method," Surf. Sci, 499, pp.15-23, 2002.

[9] T. Hikihara, "Dynamics of Single Atoms and Molecules Manipulated at Material Surface", International Symposium on Nonlinear Theory and its Applications (NOLTA2007), , HVancouver, Canada, September 17-19 (2007).

[10] T. Hikihara and K. Yamasue, "A Numerical Study on Suspension of Molecules by Microcantilever Probe", The Fifth International Symposium on Linear Drives for Industry Applications (LDIA2005), Awaji, Hyogo, Japan, September 25-28 (2005).

[11] J. A. Beswick, G. Delgado-Barrio, and J. Jortner, "Vibrational predissociation lifetimes of the van der Waals molecule $\mathrm{HeI}_{2}$," J. Chem. Phys., 70(8), pp.3895-3901, 1979.

[12] R. J. Wolf and W. L. Hase, "Quasiperiodic trajectories for a multidimensional anharmonic classical Hamiltonian excited above the unimolecular threshold," J. Chem. Phys., 73(8), pp.3779-3789, 2006.

[13] R. M. Hedges and W. P. Reinhardt, "Classical and quantum dynamics of long lived doubly excited vibrational states of triatomic molecules," J. Chem. Phys., 78(6), pp.3964-3975, 1983.

[14] S. K. Gray, S. A. Rice, and D. W. Noid, "The classical mechanics of vibrational predissociation: A model based study of phase space structure and its influence on fragmentation rates," J. Chem. Phys., 84(7), pp.3745-3752, 1986.

[15] M. Toda, "Crisis in Chaotic Scattering of a Highly Excited van der Waals Complex," Phy. Rev. Lett., 74(14), pp.2670-2673, 1995.

[16] M. O. Allen, "Introduction to Molecular Dynamics Simulation," in Computational Soft Matter: From Synthetic Polymers to Proteins, Lecture Notes, NIC Series, 23, ISBN 3-00-012641-4, pp.1-28, 2004.

[17] D. Vainchtein and I. Mexic̀, "Capture into Resonance: A Method for Efficient Control," Phy. Rev. Lett., 93, 084301, 2004.

[18] B. V. Chirikov, "A Universal Instability of Many-Dimensional Oscillator Systems," Phy. Rep., 52, pp.263-379, 1979.

[19] H. Yamada, Kyoto University, private communication (2007).

[20] V. Caciuc, H. Hölscher, adn S. Blügel, "Ab initio investigation of noncontact atomic force microscopy tip-surface instability in $\operatorname{InAs}(110)$ surface," Phy. Rev. B, 72, 035423, 2005.

[21] K. Yamasue and T. Hikihara, "Control of microcantilevers in dynamic force microscopy using time delayed feedback," Rev. Sci. Instrum., 77(5), 053703, 2006.

[22] T.Hikihara, "Dynamics in manipulation and actuation of nanoparticles", International Conference on Applications in Nonlinear Dynamics, Polpu Beach, Koloa, Hawai, Sept.24-27 (2007). 